\title{
Detection of Microorganisms with an Electronic Nose for Application under Microgravity Conditions
}

Ulrich Reidt ${ }^{1}$, Andreas Helwig ${ }^{2}$, Gerhard Müller ${ }^{2}$, Joachim Lenic ${ }^{3}$, Jan Grosser $^{3}$, Viktor Fetter ${ }^{1}$, Andrei Kornienko ${ }^{1}$, Sergey Kharin $^{4}$, Natalia Novikova ${ }^{4}$, Thomas Hummel

${ }^{1}$ Airbus Defence and Space GmbH - Space Systems, Department of TESXS Science Engineering, Friedrichshafen, Germany; ${ }^{2}$ Airbus, Central Research and Technology, Ottobrunn, Germany; ${ }^{3}$ German Aerospace Center (DLR), Bonn, Germany; ${ }^{4}$ State Scientific Center of the Russian Federation - Institute of Biomedical Problems (IBMP) of the Russian Academy of Sciences, Moscow, Russia

Abstract

In this work, we report on the construction, training and functional assessment of an electronic nose (called ' $E$-Nose') that is capable of monitoring the microbial contamination onboard space ships under microgravity conditions. To this end, a commercial electronic nose was modified to allow for the sampling of microbial volatile organic compounds (MVOCs) emitted from relevant bacterial and fungi species. Training of the modified 'E-Nose' was performed by establishing an MVOC database consisting of two Gram-positive bacteria strains (Bacillus subtilis and Staphylococcus warneri) and two fungi strains (Aspergillus versicolor and Penicillium expansum). All these strains are known to exist onboard the International Space Station (ISS) and to form important parts of its microbial contamination. All cultures were grown on four kinds of structural materials also in use onboard the ISS. The MVOCs emitted during the different growth phases of these cultures were monitored with an array of ten different metal oxide gas sensors inside the 'E-Nose'. Principal component analysis of the array data revealed that B. subtilis and S. warneri form separate clusters in an optimized score plot, while the two fungi strains of $A$. versicolor and $P$. expansum form a large common cluster, well discriminated against to the bacteria clusters.

Keywords

Sensor system $•$ Electronic nose $・$ International Space Station $・$ Space $・$ Fungi $・$ Bacteria

\section{INTRODUCTION}

The idea of developing electronic noses based on arrays of cross-sensitive sensors is more than 30 years old (Persaud and Dodd, 1982). Today, electronic noses are being used as olfactory detection systems in diverse fields of application ranging from agriculture, food and beverage industries, cosmetics, medicine, industrial quality control, military, and science (Wilson and Baietto, 2009; Alam and Saeed, 2013). In the food and beverage industry, for example, electronic noses are being used to determine the state of maturation of fruits and vegetables or to control the quality of milk and meat products (Schaller et al., 1998). Other applications concern the roasting of coffee and the classification of coffee into different brands. Electronic noses in this realm have partly replaced and partly augmented the more expensive and subjective 'Cup Tests' by human experts (Juan Rodríguez et al., 2009). A newly emerging field of research is the analysis of breath, sweat, skin, urine, sputum and blood samples of human beings to diagnose metabolic or microbiological disorders. Breath gas analysis, in particular, has made great progress in the rapid diagnosis of lung cancer and diabetes (Taivans et al., 2014; Wang et al., 1997; Dalton et al., 2004). Other activities in the biomedical field concern the detection of human pathogenic microorganisms such as Pseudomonas aeruginosa, Escherichia coli, Klebsiella pneumoniae, Helicobacter pylori, Enterobacter aerogenes, Proteus vulgaris, Staphylococcus aureus, Streptococcus pneumoniae, Enterococcus faecalis, Candida albicans, and Aspergillus fumigatus. Electronic noses detect such microorganisms via their excreted odorants, which are collectively called 'microbial volatile organic compounds' (MVOCs). The most representative groups of compounds are hydrocarbons (e.g., octane), terpenes (e.g., isoprene, geosmin), alcohols (e.g., 1-Octen-3-ol), carboxylic acids, ketones (e.g., 2-butanone), sulfur derivatives (e.g., dimethyldisulfide), aromatic compounds (e.g., styrene) and nitrogen containing heterocycles (e.g., 3-methylfuran; Wilkins et al., 2000). 
Most of these compounds can be sensitively detected with the help of solid-state gas sensors (Korpi et al., 1998; Korpi et al., 2009). Usually, such sensors exhibit a wide spectrum of cross sensitivities, which however depend on the choice of sensor materials and sensor operating conditions. Forming arrays of several such sensors and by making proper choices of sensors and sensor operating conditions, such arrays can produce multi-dimensional data vectors, which can be regarded as fingerprints pointing to specific microorganisms. A good example for an electronic detection of microorganisms is the so-called 'MonoNose'. This electronic nose was used for the identification of several pathogenic bacterial strains originating from clinical samples. The identification of MVOCs with this electronic nose was in real time and succeeded to differentiate several different bacterial strains such as $E$. coli, Klebsiella oxytoca, S. aureus, $E$. faecalis and $P$. aeruginosa (Bruins et al., 2009). A second example of an electronic nose, which was used for the detection and identification of microorganisms, is the 'Cyranose 320'. This system was able to detect $S$. aureus, Haemophilus influenzae, Streptococcus pneumonia, Moraxella catarrhalis and the two famous bacteria strains $E$. coli and $P$. aeruginosa. Applying 'principal component analysis' (PCA), it was possible to separate the smell signals from each bacteria strain into clearly distinguishable clusters (Dutta et al., 2002). Both examples demonstrate the potential and the capabilities of electronic noses to detecting and identifying microorganisms.

In this paper, we report on the construction, training, and commissioning of an electronic nose for the detection of microorganisms inside the 'International Space Station' (ISS). The aim of this research is to provide a sensitive detection system for bacteria and fungi that is able to work under microgravity conditions in space and that can provide data on the microbial contamination inside the ISS without necessitating space transport of swabs down to earth and off-site analysis in earth-bound laboratories. Such onboard detection systems must satisfy a number of specific requirements such as easy handling, liquid- and label-free operation, and measurement results in real time. In order to arrive at this goal, a commercial 'E-Nose' was modified to allow for the sampling and analysis of MVOCs, emerging from microorganisms. In order to enable the 'E-Nose' for its intended application in space, four different microorganisms, which were known to be present onboard the ISS, were cultured and grown on structural materials also used inside the ISS. With this MVOC database, the 'E-Nose' was trained and the detection algorithms were optimized to fulfill the intended detection task. As a key result, we found that the two fungi strains Aspergillus versicolor and Penicillium expansum and the two Gram-positive bacterial strains Bacillus subtilis and Staphylococcus warneri can be sensitively detected and discriminated against the two fungi strains of $A$. versicolor and $P$. expansum by a straightforward PCA.

\section{MATERIALS AND METHODS}

\section{Strains and Cultivation of Microorganisms}

For building up an MVOC database, two bacteria and two fungi strains were selected that were known to be present onboard the ISS. To this end, the bacterial strains Bacillus subtilis (BS) (DSM No.: 1088) and Staphylococcus warneri (SW) (DSM No.: 20316) as well as the fungal strains Penicillium expansum (PE) (DSM No.: 1282) and Aspergillus versicolor (AV) (DSM No.: 1943) were first cultivated from a freezedried stock culture obtained from the Leibniz Institut DSMZDeutsche Sammlung von Mikroorganismen und Zellkulturen $\mathrm{GmbH}$ in Braunschweig, Germany. After the first growth, both bacterial strains were cultivated with Tryptic Soy Broth (TSB) medium and fungal strains were grown at Czapek-Dox (CZ) agar and Czapek-Dox broth medium received from SigmaAldrich Chemie $\mathrm{GmbH}$, Munich, Germany. The dehydrated powders from all media were suspended in $1 \mathrm{I}$ of distilled water and were sterilized at $121^{\circ} \mathrm{C}$ for $15 \mathrm{~min}$. After sterilization and cooling down to $45-50^{\circ} \mathrm{C}$, the media were poured into Petri dishes. For gas-sensing experiments, $2-5 \mathrm{ml}$ of each culturing medium was transferred to mini Petri dishes. Subsequently, the mini Petri dishes were placed into custom-built gas vessels, which allowed the emerging cultures to grow under controlled gaseous environments and temperature inside a cultivation station (Figure 1). For negative control measurements, pure media without microorganisms were used. In order to ensure identical growth conditions for all cultures, a constant flow of $1 \mathrm{l} / \mathrm{min}$ of humidified synthetic air (SA; rH $=70-90 \%)$ was guided through all mini Petri dishes. Additionally, some of the cultures were grown on materials that were being used inside the ISS. To these cultures, identically sized samples of sterilized materials such as Nomex, cable marker, aluminum, and printed circuit board (PCB) were added to the culturing medium (Figure 2). After inoculation, all cultures were cultivated at an ambient temperature of $20^{\circ} \mathrm{C}$, over periods of time ranging from $24 \mathrm{~h}$ up to 14 days. As these cultures progressed through different growth phases, samples were taken for measurements using the 'E-Nose'.

After measurements, the colony forming units (CFUs) were determined only from the bacterial samples. Lower bacterial cell concentrations were obtained by drying out the cultures inside the cultivation station, leading to selectively dehydrated cultures. During this drying-out procedure, between 70 and $150 \mathrm{~h}$, the CFUs progressively decreased. To determine the CFUs from a dried-out culture, $1 \mathrm{ml}$ of sterile water was added to the mini Petri dish containing the dried-out culture and the encrustation was dissolved to form a homogeneous solution. Thereafter, these cultures were processed as normal liquid bacterial cultures. For liquid bacterial cultures, a dilution series was made ranging from undiluted to $1: 10^{6}$, and $100 \mathrm{ml}$ from 
A)

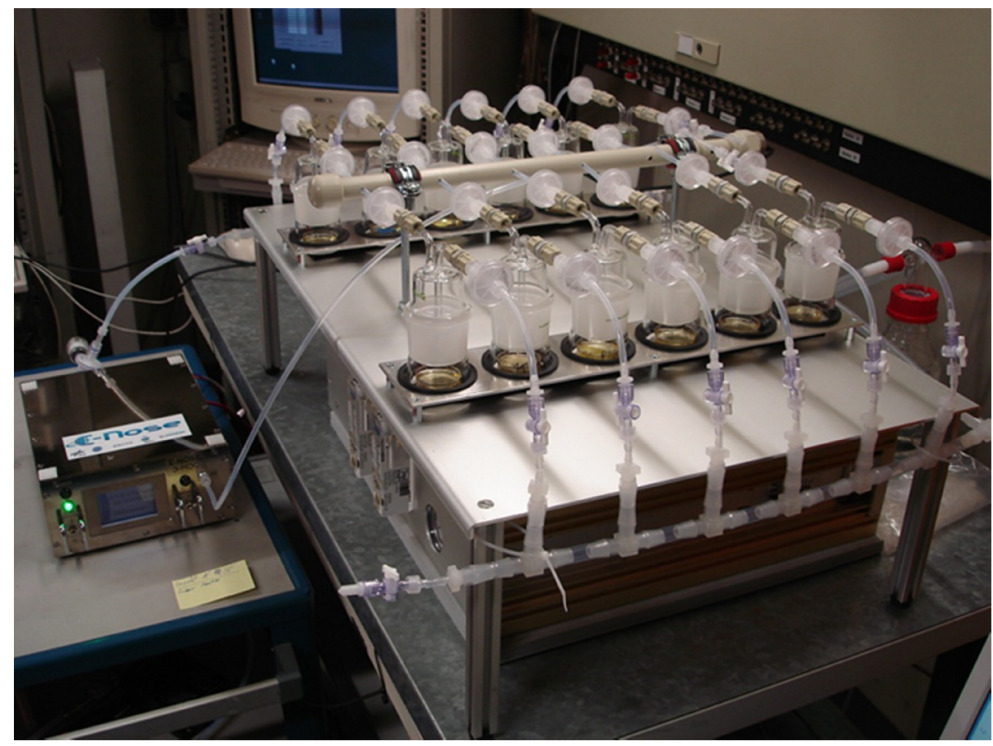

B)

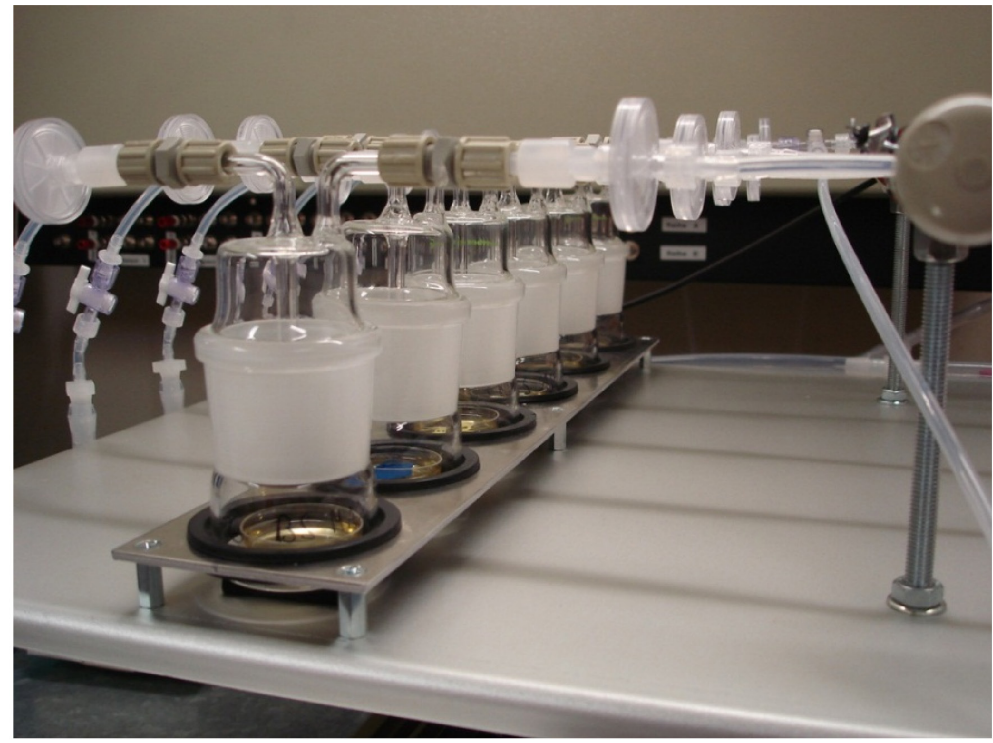

Figure 1. (A) Cultivation station with twelve custom-built gas vessels in which the organisms are grown under controlled gaseous environments. Left hand side the E-Nose' is contacted for growth monitoring of the cultures. (B) Gas vessels with the culture of Bacillus subtilis in liquid Tryptic Soy Broth media.

each dilution was streaked onto Tryptic Soy Agar. The culture plates were incubated at $30-37^{\circ} \mathrm{C}$ overnight, and the number of CFUs was determined at the end of the incubation time.

\section{Design of 'E-Nose' System and Air Sampler}

The MVOCs emerging from the different cultures were monitored with the 'E-Nose' system containing an array of ten different kinds of metal oxide (MOX) gas sensors. The sensor system containing this array is shown in Figure 3, featuring in addition a sampling head called 'Air Sampler', which was constructed to acquire the MVOCs from the microbiological cultures. In order to fulfill the specific task of air sampling in space, e.g., at the ISS, all sensors in the array were chosen to exhibit cross-sensitivity profiles with peak sensitivities 


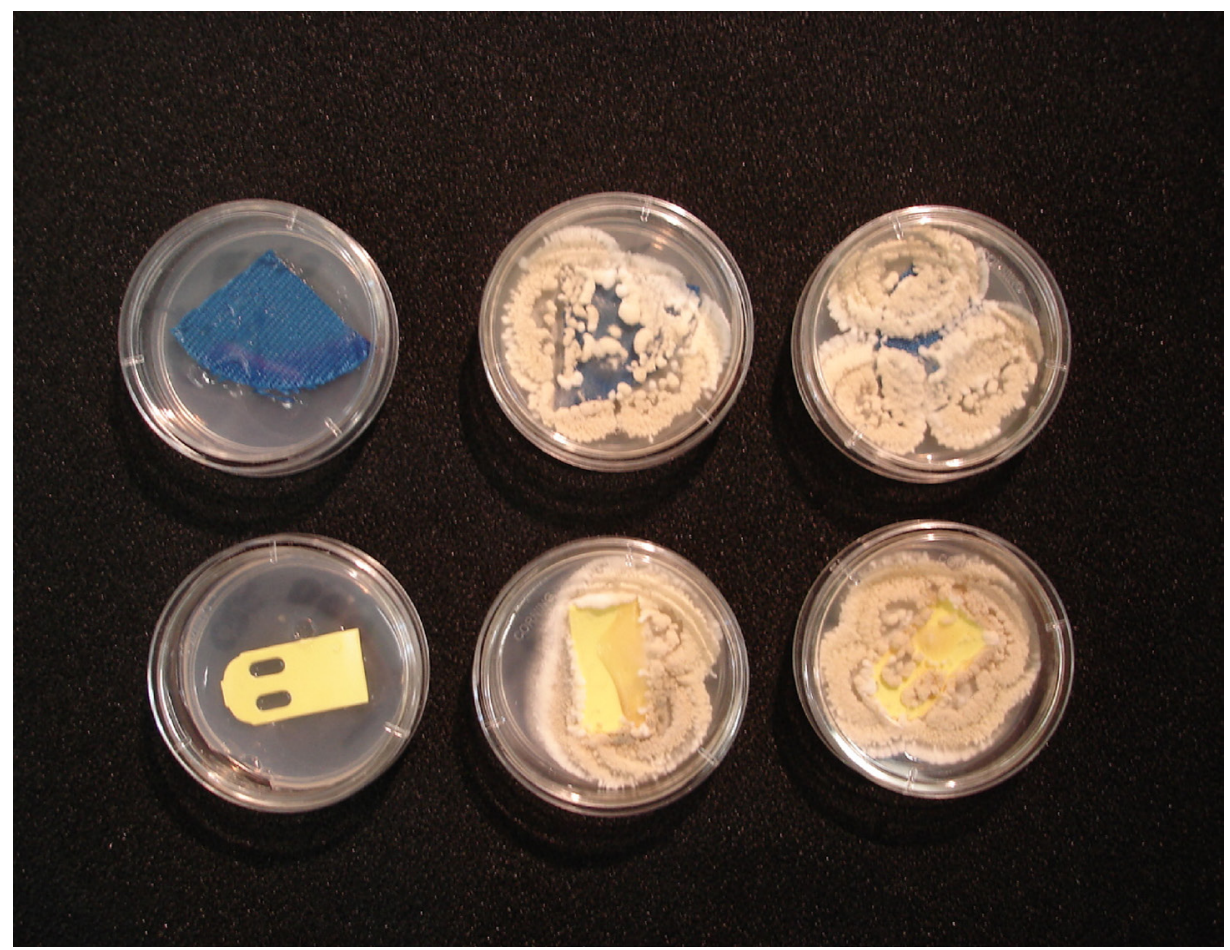

Figure 2. Fungi culture of Penicilium expansum growing on the substrates NOMEX (top line in blue) and cable marker (below line in yellow) at mini Petri dishes, left hand side the negative control without Penicilium expansum.

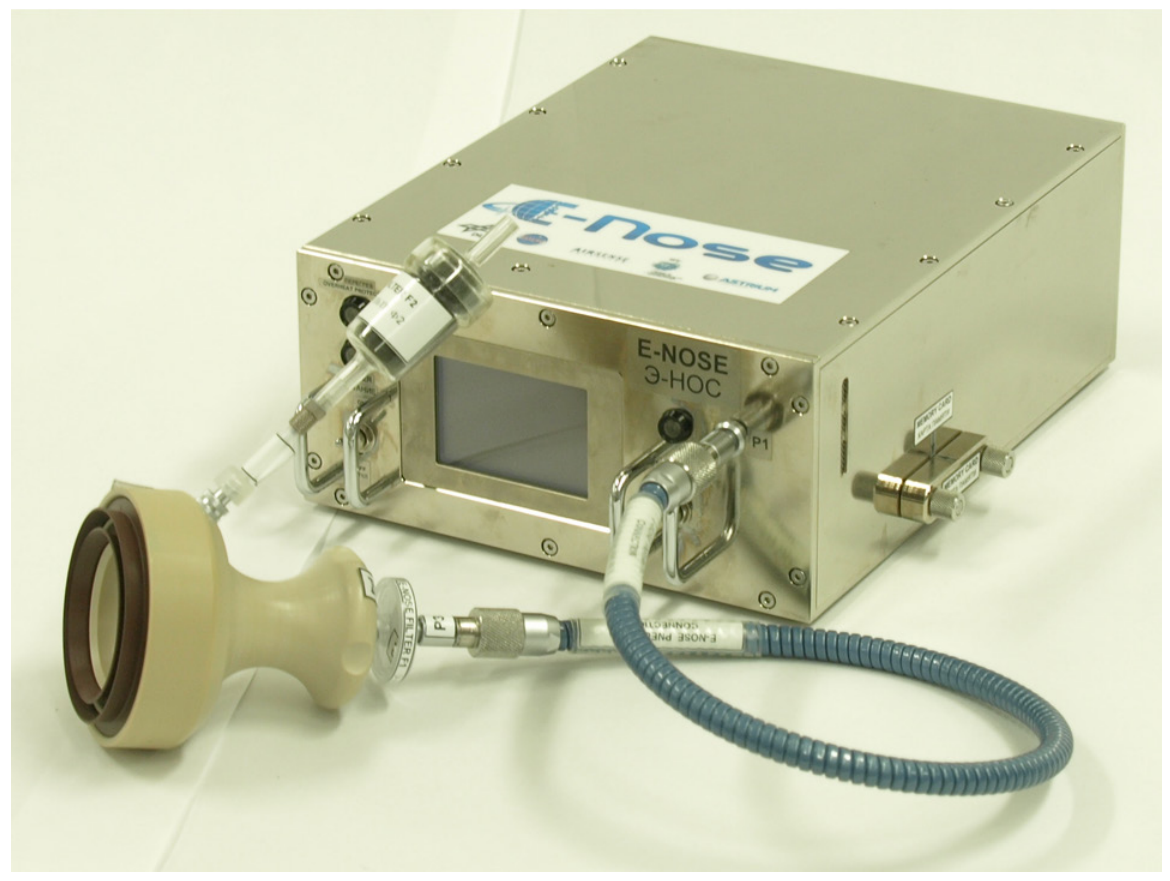

Figure 3. 'E-Nose' system featuring a display for programming and data visualization with external sampling head ('Air Sampler') for taking MVOC air samples from microorganisms. 


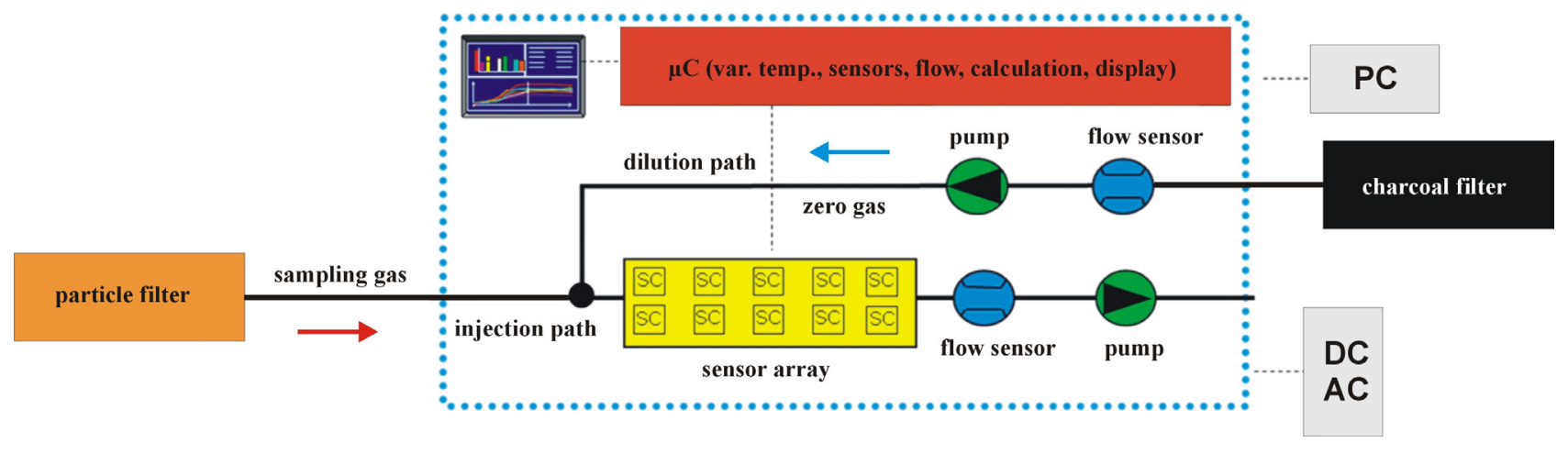

Figure 4. Block circuit diagram of the 'E-Nose'.

matching dominant MVOC species emerging from the abovedescribed cultures. All sensors were purchased from UST, Germany, and operated according to their specific supplier recommendations. For convenience, a list of the sensors and their corresponding recommended chemical compound sensitivities is presented in Table 1 (Su et al., 2014; Baietto et al., 2010). As shown in the block circuit diagram of Figure 4, the sensor array was constantly exposed to a stream of humidified synthetic air (SA). This background SA was supplied from gas cylinders containing high-purity SA (Linde, Germany; 80(79)\% $\mathrm{N}_{2}, 20(21) \% \mathrm{O}_{2}$ ). In order to avoid chemical cross-talk in between neighboring sensors, a minimum distance of $10 \mathrm{~mm}$ was maintained between neighboring sensors. In the interest of a fast sensor reaction, the flow of the carrier gas was relatively high, enabling a tenfold exchange of the air volume inside the sensor chamber per minute. In order to enable sensing, the gas mix emitted from the cultures and extracted through the sampling port was admixed to the background air stream. This sampling air stream was generated by a small pump positioned downstream of the sensor array. In the interest of stable sensor operating conditions, the flow rate of the sampling air stream was always kept at $400 \mathrm{sccm}$. In order to acquire gas sensor signals, the resistances of the sensing layers were monitored using a commercial 12-bit data acquisition board. In addition to the data acquisition, this board also allowed the substrate heater meanders of the individual sensors to be powered and their temperatures to be controlled. The entire system including sensors, data acquisition board and pumps was controlled by custom-designed software. This program also drove the small display on the 'E-Nose' system, which served the dual purpose of a man-machine interface and a data display. Central to the functioning of the entire system is the method of air sampling from the cell cultures. The functioning of the sampling port is shown in a higher level of detail in the CAD drawing of Figure 5. The sampling head ('Air Sampler') consists of a turned piece of polyetheretherketone with a central opening on the bottom, which can be fitted over
Table 1: Sensors and chemical compounds sensitivity from the 'E-Nose'.

\begin{tabular}{lc}
\hline No. of sensors of the 'E-Nose' and chemical compounds sensitivity \\
\hline $\begin{array}{l}\text { Sensor } \\
\text { 1: }\end{array}$ & Aromatic compound, Alkane \\
Sensor & Nitrogen oxides, Alcohols, Aromatic compounds \\
2: & Aromatic compound, Alkane \\
Sensor & Hydrogen \\
3: & \\
Sensor & \\
4: & Aromatic compounds, Alkane \\
Sensor & \\
5: & Methane, Alcohol \\
Sensor & \\
6: & Inorganic sulfur compounds, Terpenes, Sulfur containing \\
Sensor & organic compounds, Aromatic compound \\
7: & Alcohol, Aromatic compound \\
Sensor & \\
8: & \\
Sensor & Aromatic compounds and inorganic sulfur compounds as $\mathrm{H}_{2} \mathrm{~S}$ \\
9: & \\
Sensor & Methane and aliphatic organic compounds \\
10: &
\end{tabular}

the cell culture plates. An O-ring around this opening allows the sampling head to be tightly positioned over the mini Petri dishes containing the microbiological cultures, thus separating them from the ambient air. Active gas sampling from the cell plates is performed by exposing the plates to a small stream of purified air $(400 \mathrm{sccm})$. After interacting with the mini Petri dish, the sampling air stream is extracted through the central bore hole in the sampling head into the 'E-Nose' system.

\section{'E-Nose' Measurements}

All gas-sensing experiments were performed with the 'Air sampler' connected to the 'E-Nose' via the transfer line. The measurement procedures themselves were controlled by the software WinMuster obtained from Airsense Analytics, 


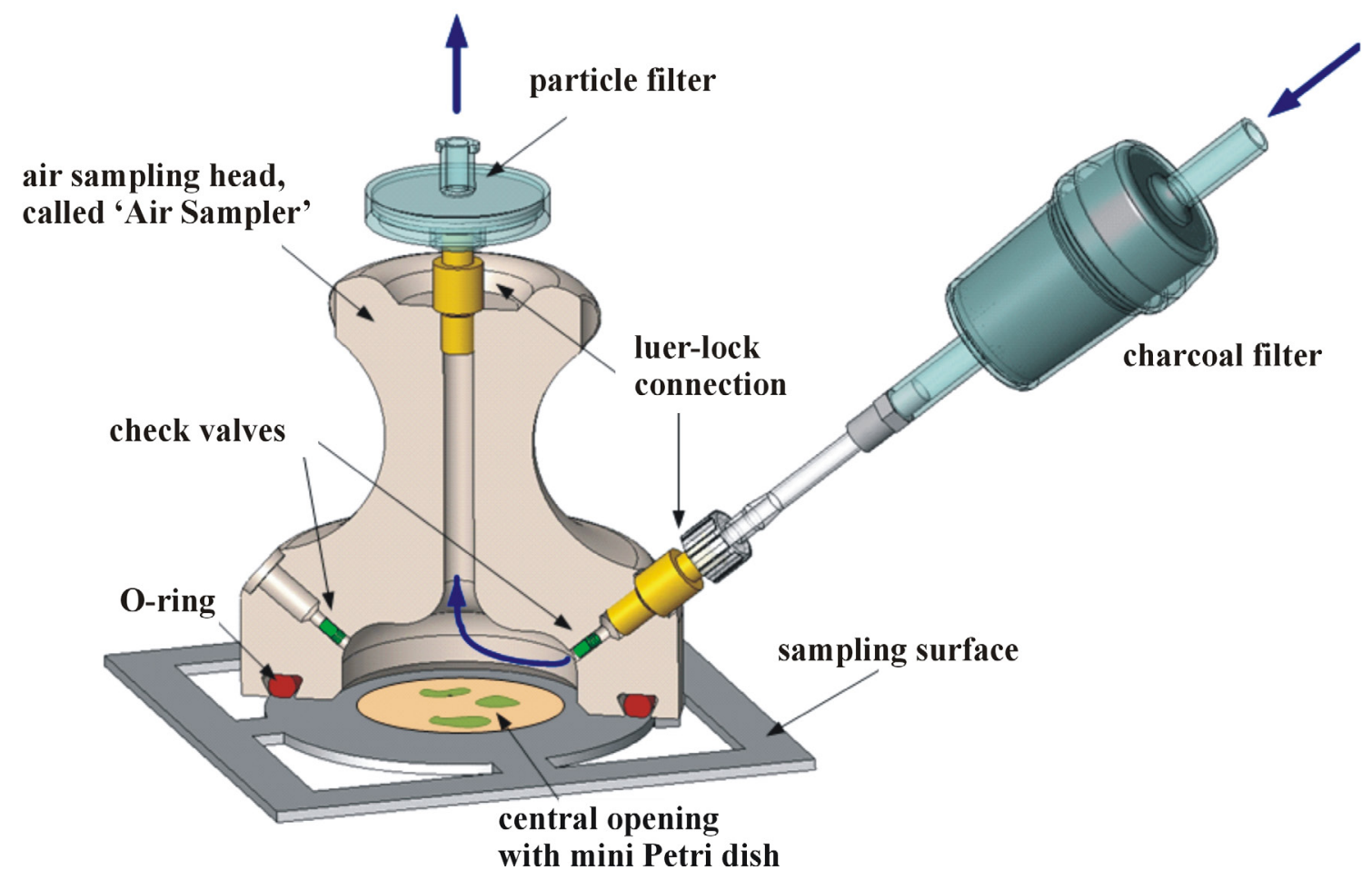

Figure 5. CAD drawing of air sampling head called 'Air Sampler'.

Germany. The data sampling rate was set to $1 \mathrm{~Hz}$. Prior to each gas measurement, a sensor cleaning procedure was performed, which was maintained for 300 s. During this cleaning step, the transfer line and the 'Air sampler' were being flushed with $200 \mathrm{sccm}$ removing the head space volume of the mini Petri dishes. Thereafter, the sensor array was exposed to the sampled gas stream for another $300 \mathrm{~s}$. The sensor chamber flow during the sensor cleaning and sampling procedures was set to $400 \mathrm{sccm}$ without any dilution during the sampling period. During each measurement, a set of 300 data points was collected, starting within the onset of the sampling air stream through the 'Air sampler'. Finally, the acquired raw data were stored on a computer for later processing.

\section{'E-Nose' Data Processing}

After the end of the measurements, all raw data were analyzed and evaluated with the software 'Origin' from OriginLab Corporation, USA. The so produced data were then processed using the software 'MATLAB' from MathWorks Corporation, USA. Final representation of the data was performed in the form of score plots derived from a PCA of the 'E-Nose' data. The PCA itself was performed using the software 'PLS_ Toolbox' (for MATLAB), obtained from Eigenvector Research Incorporated, USA.

\section{RESULTS}

Monitoring of Bacterial Growth from B. subtilis by the 'ENose'

To study the bacterial growth of viable cells, bacteria should be inoculated in sterile broth media and the culture incubated under optimal growth conditions. The bacteria start with cell division and cause the cellular mass to increase. The dynamics of the bacterial growth can be studied by plotting the cell growth versus the incubation time of the culture. The obtained curve follows a sigmoid curve and is known as the standard curve of bacterial growth. Normally, the increase in the cell mass is measured by monitoring the optical density at a wavelength of $600 \mathrm{~nm}$ with the use of a photometer. The degree of turbidity in the media is directly related to the bacterial growth of microorganisms in the culture. With this background in mind, the time evolution of bacterial growth can be subdivided into four phases: the culture starts first with a lag phase (1) were the bacteria adapt to their environmental conditions such as nutrients, temperature and $\mathrm{pH}$ value. After this first phase, an exponential growth phase (2) follows where the growth of the bacteria is not limited and in which cell doubling takes place at a constant rate. This exponential growth phase is followed by a stationary phase (3) in which 
the growth rates and the death rates are balanced due to the limitation of growth factors and inhibitory substances produced by the bacteria themselves. Finally, the growth ends with the death phase (4). In this final phase, the bacteria modify their environment in such an extreme way that no life is anymore possible; accordingly, the number of dead cells exceeds the number of living cells and the culture decays. In this study, we abandon the standard optical analysis of the bacterial growth and replace it by 'E-Nose' monitoring. The bacterial strain $B$. subtilis was cultured in glass vessels, which were placed in a cultivation station for optimal growth conditions. The 'E-Nose' was connected to the culture by a transfer line, and the MVOCs emitted from the culture were monitored. As shown in Figure 6, only the signals of sensor 7 were used to represent a growth curve of $B$. subtilis because sensor 7 responded with the highest amplitude of all sensors. The growth curve started with the lag phase where only a few MVOCs were produced by the bacteria and where the signals only slightly exceeded those of the negative control, which was TSB medium without bacteria. As the bacteria entered the exponential phase, the emission of MVOCs strongly increased. Thereafter, the culture progressed into its stationary phase and the response of sensor 7 saturated. Finally, the culture was drying out and entered its death phase. In this final phase, the sensor 7 response decreased and approached the level of the negative control again. To better illustrate the reduction of the bacteria in the death phase, the CFU was determined at two different positions in the monitoring process. One measurement was taken in the decreasing region of the curve and the other at the end of the gas measurement cycle where the signal had decreased to the low level of the negative control. In the first case, a CFU of $1.3^{\prime} 10^{6} \mathrm{cells} / \mathrm{ml}$ was determined and in the latter, a CFU of $7.6^{\prime} 10^{4} \mathrm{cells} / \mathrm{ml}$.

\section{Comparison of Growth between B. subtilis and S. warneri Monitored with the 'E-Nose'}

Two bacterial strains were used to build up an odor database for the 'E-Nose'. However, first of all the growth behavior of the strains $B$. subtilis and $S$. warneriwas investigated. Both bacteria strains were inoculated with the same bacteria concentration and the same volume of culturing media. The MVOCs emerging from the individual cultures were then measured, one by one, by monitoring the response of sensor 7 of the 'E-Nose'. As negative control, the culture medium (TSB) was used without bacteria to detect the background odor of the medium. To avoid measurement inaccuracy, double measurements were performed for each bacterial strain. In the exponential phase of the cultures, both strains showed nearly the same growth behavior. However, after the changeover from the exponential to the stationary phase, S. warneri produced more smell, caused by a higher cell number in the respective culture, and the resulting sensor signals clearly exceeded those produced by $B$. subtilis. Thereafter, the cultures started to dry out and the death phase was initiated. Whereas in this final phase, $B$. subtilis still produced stable signals with a constant amplitude, S. warneri produced rising signals at sensor 7 (Figure 7). In this regard, both bacterial strains showed distinctly different growth behaviors. Considering the phylogeny and the natural environments of both bacteria this is not surprising as $B$. subtilis is a classical environmental bacterium, which is very robust against environmental influences such as dryness, whereas $S$. warneri is a commensal of the human skin and therefore more sensitive to dehydration. This difference is clearly reflected by the MVOC emissions in the death phase of both bacterial strains.

\section{Measurements of Fungi Cultures from $A$. versicolor and $P$. expansum by the 'E-Nose'}

In addition to the two bacteria strains discussed above, the two fungi strains $A$. versicolor and $P$. expansum were analyzed by the 'E-Nose'. Both fungi were cultured on liquid media to analyze the early emissions of MVOCs from the organisms and to see which sensors respond to their typical smells. As a negative control, culture media without fungi were used to determine the background smell of the media. The experiments started with an inoculation of the medium with the same spore concentration of $870 \mathrm{CFU} / \mathrm{ml}$ for each strain. Cultures were then investigated with the 'E-Nose', and after $72 \mathrm{~h}$, the first growth of fungi was visible and first sensor signals could be observed. Starting from this point of analysis, three out of ten sensors showed a response to both kinds of fungi and the same sensors dominated. In both cases, sensors 6,7 and 8 showed higher signals than after exposure to the negative control. As only the amplitudes of the three sensors differed, a discrimination of both fungi strains is not possible with the currently used sensor array (Figure 8). The result shows that both fungi strains probably emit nearly the same MVOCs.

\section{'E-Nose' Measurements of Four Selected Organisms on Four Different Materials}

For the training of the 'E-Nose', all four microorganisms were cultivated on four different materials used within the ISS. In detail, all microorganisms were cultivated at Nomex, nonanodized aluminum, printed circuit board, and cable labeling material (Figure 2). For sample preparation, the fungi were cultivated together with sterile materials at agar media to overgrow the substrates. In the case of the bacteria, the materials were stored in the liquid media used for culturing. As negative controls, two media were measured: first, ambient air acquired through an empty 'Air Sampler', and second, culture media (TSB and Czapek-Dox) without organisms. Furthermore, it was ensured that both materials had no inherent odor and no growth-inhibiting effects (data 
A)

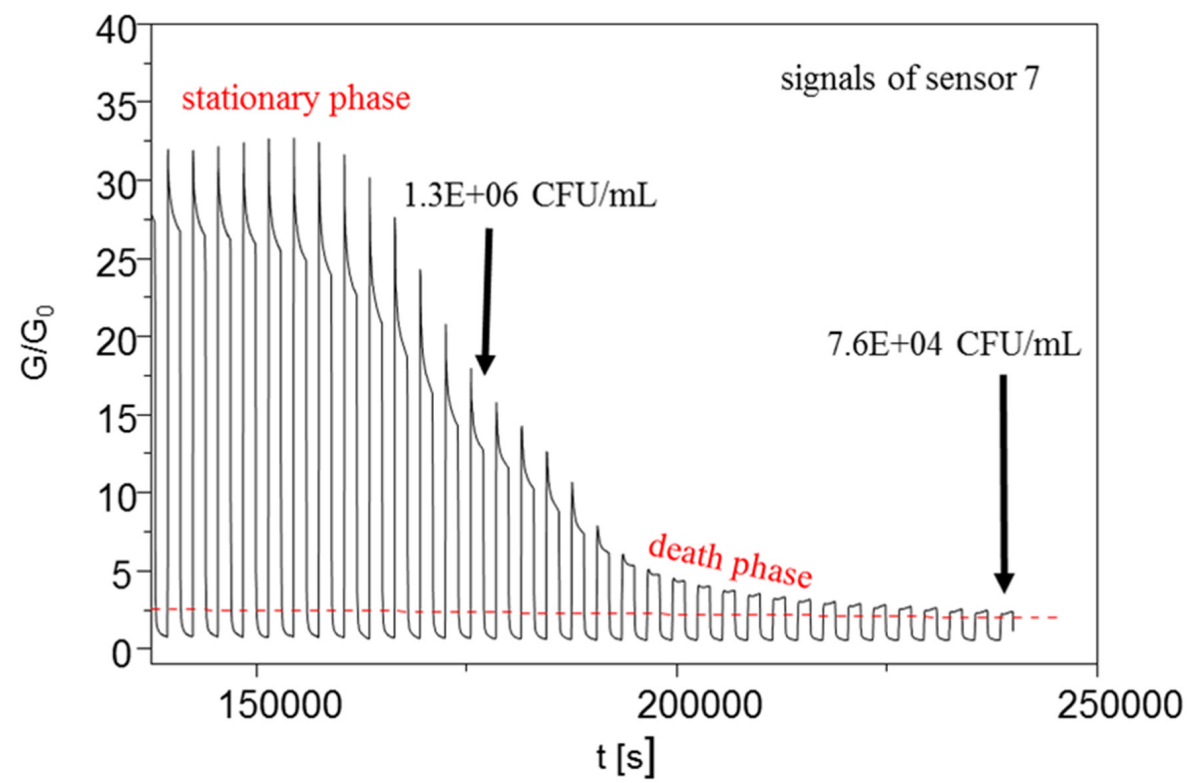

B)

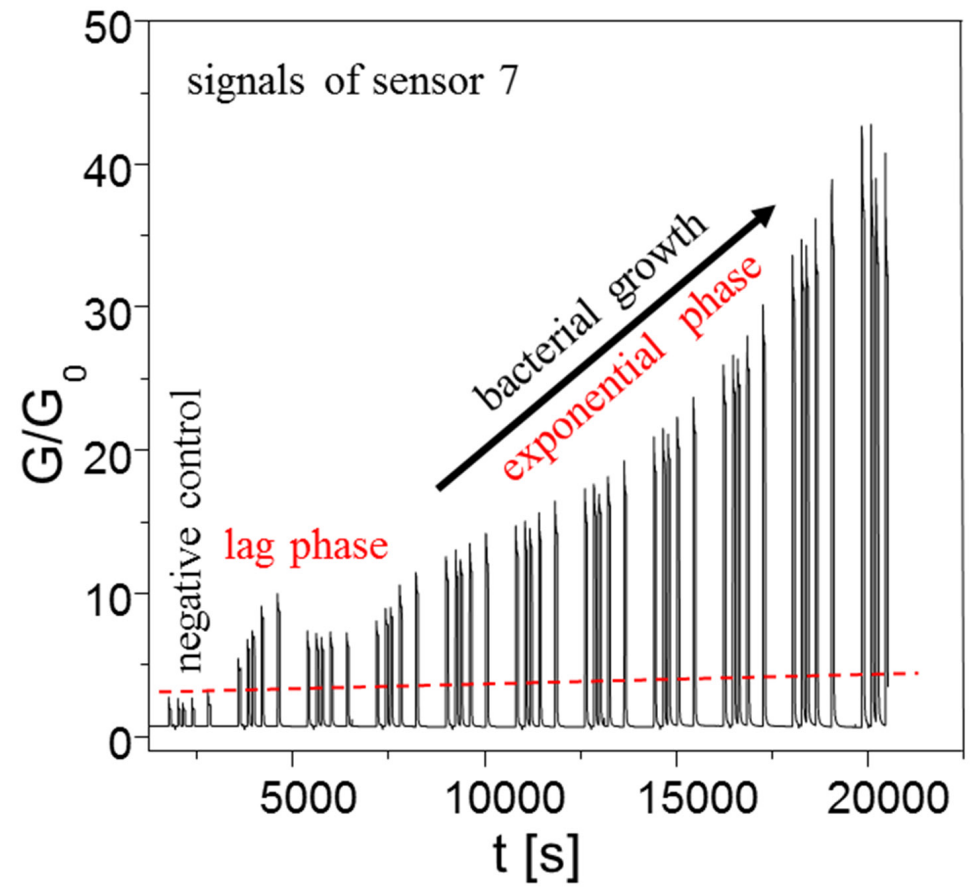

Figure 6. Bacterial growth from Bacillus subtilis as monitored by sensor 7 of the 'E-Nose'. (A) Initiation of bacterial growth after an initial lag phase followed by exponential growth; results of negative control experiment (culture media without bacteria) are shown for comparison, (B) stationary and death phases of the same culture including two CFU determinations. The red dotted line indicates the sensor response towards the negative control. 


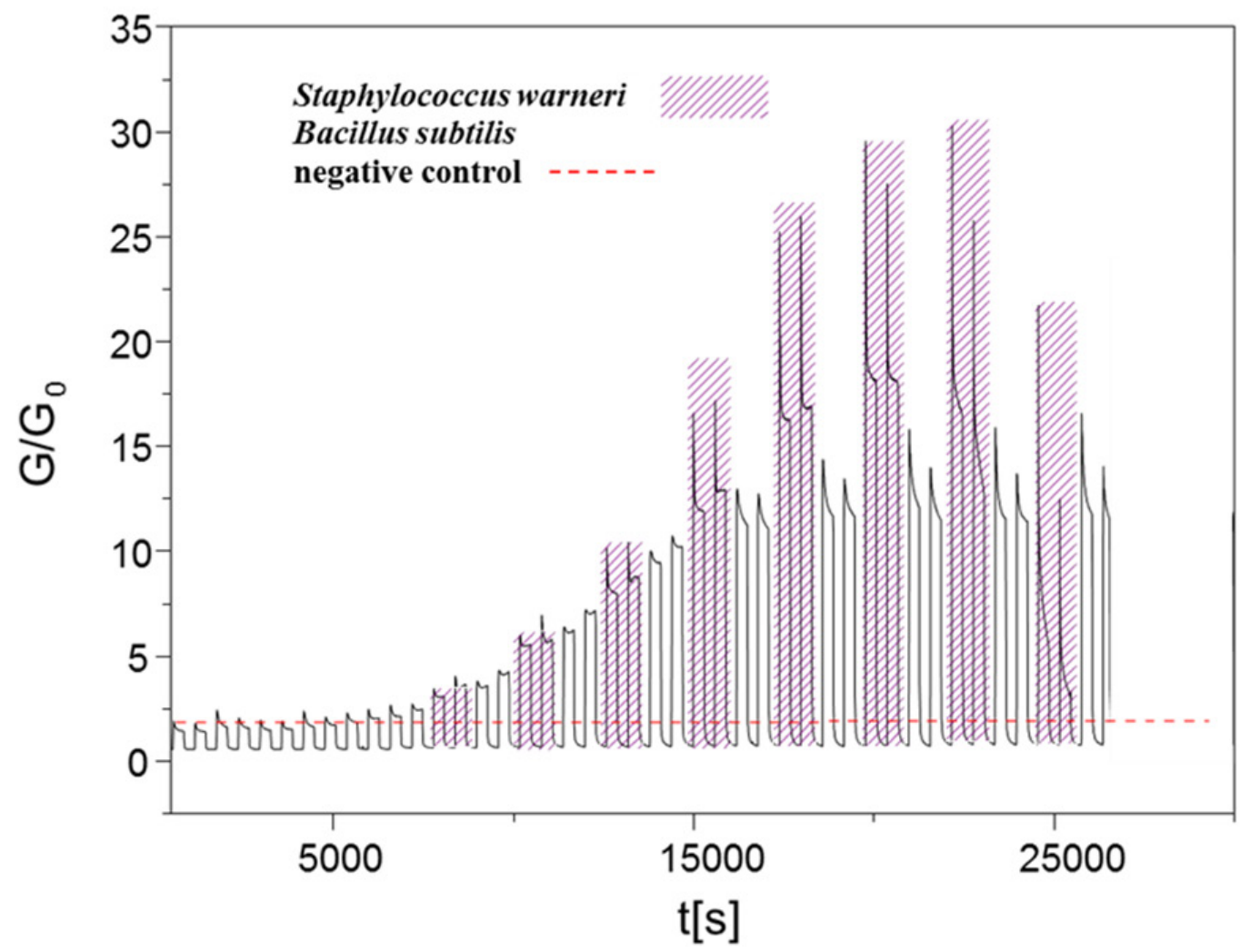

Figure 7. Growth of Bacillus subtilis and Staphylococcus warneri as monitored by sensor 7. Hatched areas: Staphylococcus warneri, non-hatched areas: Bacillus subtilis. The signal amplitude corresponding to the negative control is represented by the red dotted line. The negative control was sterile culture media without bacteria.

not shown). At each growth phase of the organisms, several gas measurements were performed by the 'E-Nose' and the negative controls were analyzed for comparison. As a first result, no influence of the materials concerning the smell of microorganisms could be observed. In other words, organisms with and without materials had the same smell. As a second common result, no changes in the odors arising in the four growth phases of both microorganisms (lag phase, exponential phase, stationary phase, and death phase) could be observed. This implies that neither the chosen materials nor the growth phases had any effect on the composition of the emitted MVOCs. After all measurements had been performed, the collected data were analyzed using PCA and displayed in the form of score plots. In these plots, both bacterial strains shine up as separate clusters, while both kinds of fungi form a single common cluster. As shown in the PC1/2 plot of Figure 9, B. subtilis (green squares) and $S$. warneri (red rhombs) are located in the top left, while both fungi ( $P$. expansum, blue triangle, and $A$. versicolor, turquoise triangle) form a common large cluster, well separated from the bacteria ones. The negative controls done with ambient air through the empty 'Air Sampler' (gray spheres) produced two small clusters close to the center of the PC1/2 score plot. These two small clusters arise from differences in the ambient humidity. The high-humidity cluster $(70-80 \%)$ is located closer to the center of the PC1/2 score plot, while the low-humidity one (30-40\%) is located closer to the bottom left of the PC1/2 plane (Figure 9). Considering the PC-2/3 score plot, the same kind of clustering can be seen. In this diagram, the fungi are localized on the left-hand side, whereas the bacterium $B$. subtilis extends to the bottom right of the plot. S. warneri differs from the former microorganisms in that it produces a cluster that extends out orthogonally from the plain of the PC-2/3 score plot forming a cluster opposite to the clusters of $B$. subtilis and the two fungi strains. In addition, the two ambient-air negative controls divide into two separate clusters depending on the humidity in the ambient air (Figure 10). For this reason, a three-dimensional PC-2/3/4 score plot was generated. In this latter illustration, the ambient-air negative controls are located in the center of the diagram, whereas all organisms form clusters extending from the origin of the $\mathrm{PC}-2 / 3 / 4$ score plot into different directions of the PC-2/3/4 
A)

B)
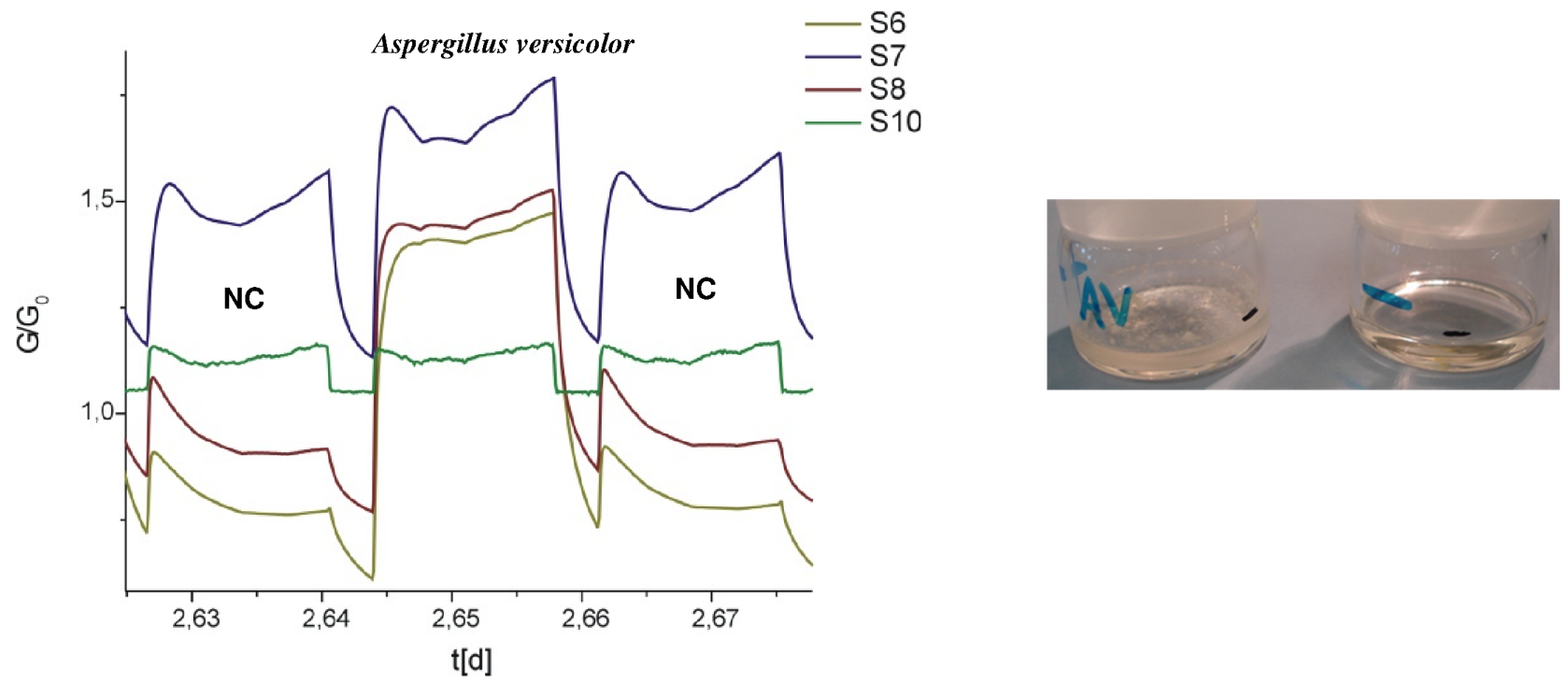

C)

D)
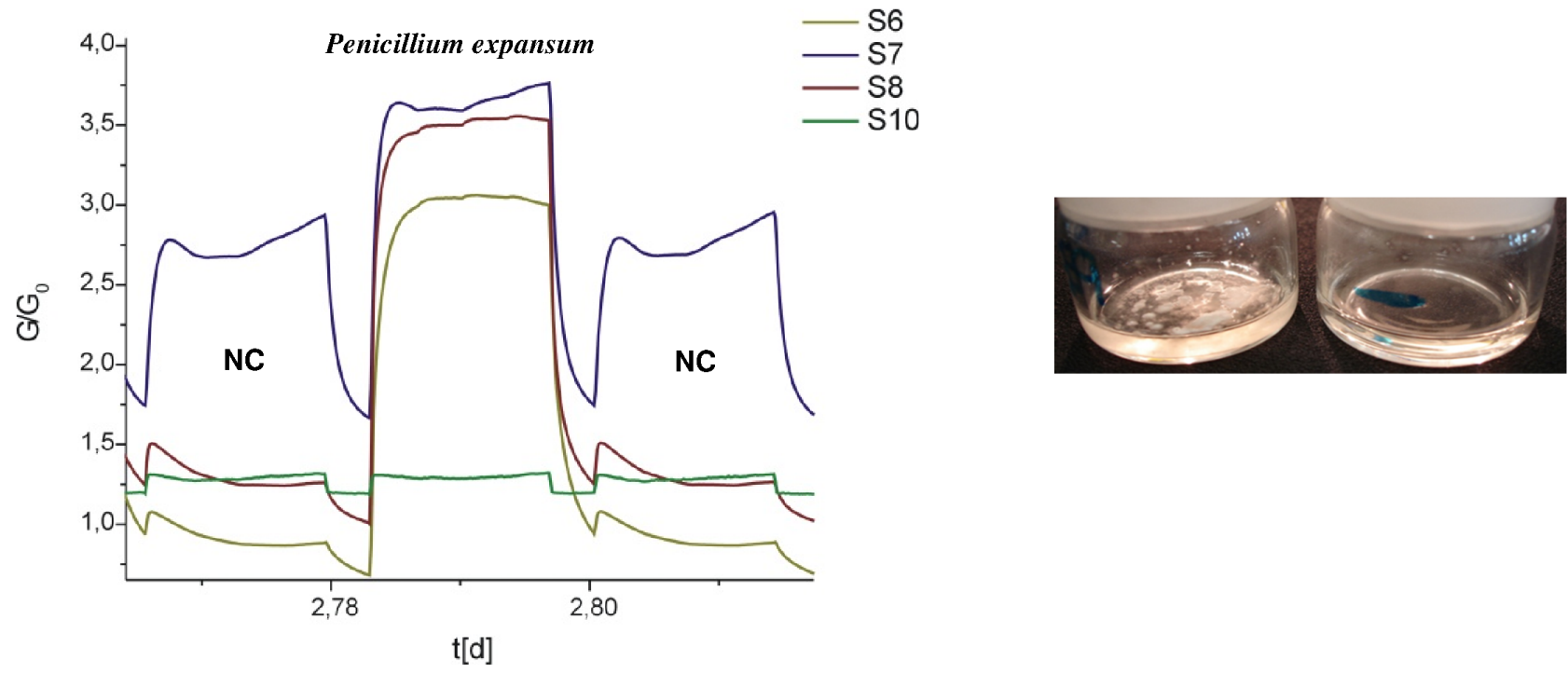

Figure 8. Early emissions from young fungi cultures. (A) Amplitudes of sensors S6, S7, S8 and S10 from Aspergillus versicolor. (B) Left: young culture of Aspergillus versicolor; right: negative control (NC), culture medium Czapek-Dox Broth without organism; (C) Responses of sensors S6, S7, S8 and S10 to Penicillium expansum; (D) Left: young culture of Penicillium expansum; right: negative control (NC), culture medium Czapek-Dox Broth without organism.

space (Figure 11). In the course of further investigations, culture media (TSB and Czapek-Dox) without organisms were measured as negative controls and all strains were calculated in relation to these negative controls. Results were also diagramed in a three-dimensional PC-2/3/4 score plot.
Signals from pure culture media were likewise located in the center of the plot, whereas the clusters of the microbial strains extended out into different directions of the PC-2/3/4 space. The bacteria strains starting with $B$. subtilis were located on the left-hand side, followed by the cluster of $\mathrm{S}$. warneri, which 


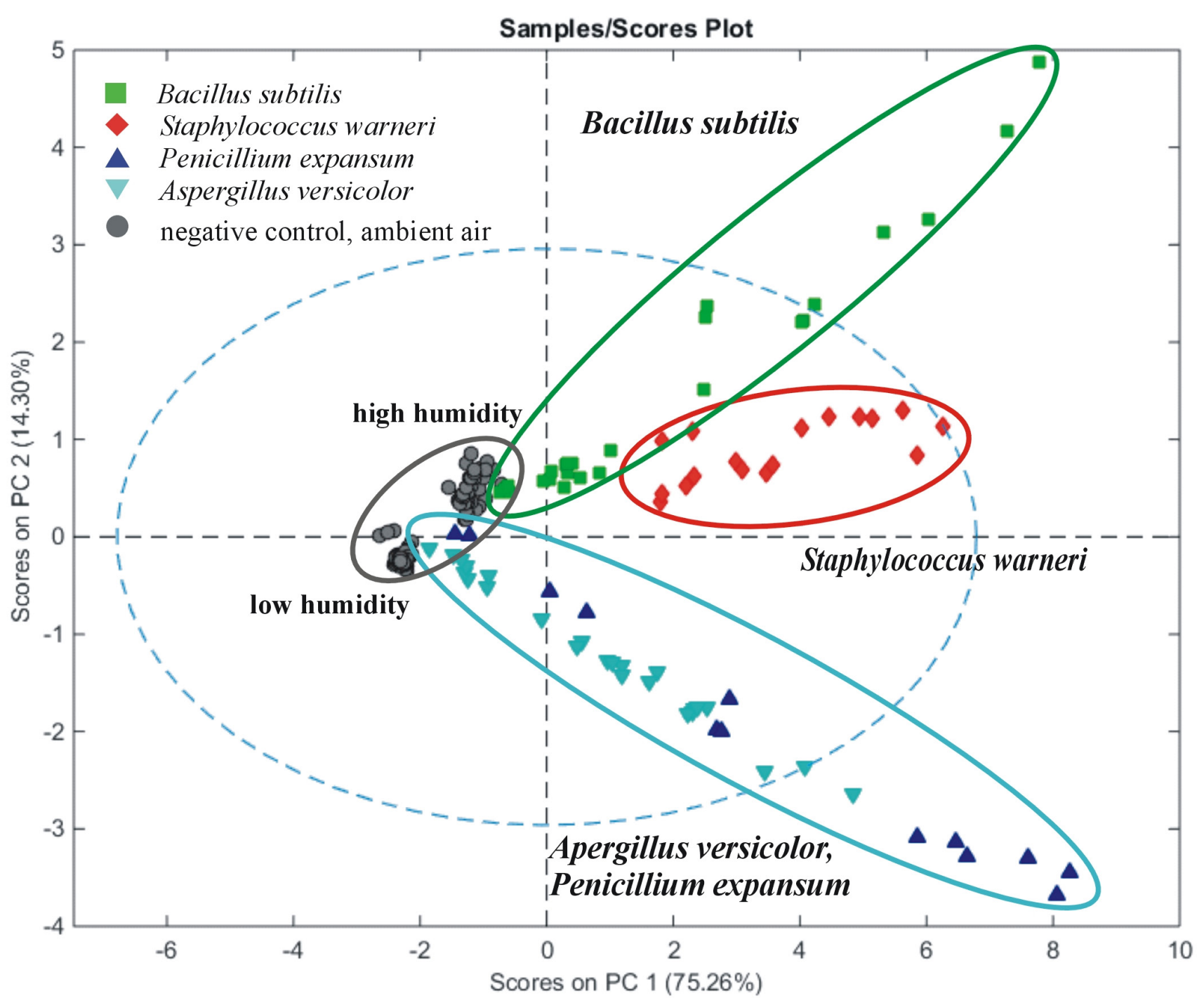

Figure 9. PC-1/2 score plot of 'E-Nose' data. Data clusters deriving from measurements on different microorganisms are indicated by colored ovals. Bacillus subtilis: green squares, Staphylococcus warneri: red rhombs, Penicillium expansum: blue triangle and Aspergillus versicolor: turquoise triangle; grey spheres: results of negative control experiments with ambient air.

was located in a position below the PC-2/3 plain. In contrast to the cocci, the common cluster of both micromycetes mapped into the PC4 direction (Figure 12). As a common result, we find that in each score plot, both bacteria strains form independent clusters, whereas both fungi only form one single cluster.

Encouraged by the PCA results that give an impression on the discrimination possibility on first sight, also the classification capability of the measured data has been analyzed. For classification purposes, a 'Partial Least Squares Discriminant Analysis' (PLSDA) was performed (Marco, 2012). The evaluated database consisted of 187 measurements in total. The portion of negative measurements, clean air sampler measurements without a sample and negative control samples of growing media TSB as well as CzapekDox (CZ) without organisms consisted of 93 measurements. The positive sample batch consisted of 41 samples of $S$. warneri and 33 samples of $B$. subtilis both grown on TSB and 20 samples of $P$. expansum and $A$. versicolor grown on $\mathrm{CZ}$. After decluttering the gathered raw data using a 'Generalized Least Squares Weighting' method and the same normalization procedure as already described earlier, the database was split in two parts with almost equal sample size (94:93). By splitting the data, it was also tried to obtain equal portions of positive and negative measurements on each dataset. The first part with 94 samples formed the calibration set and was used to build the PLSDA model. The model consisted of five latent 


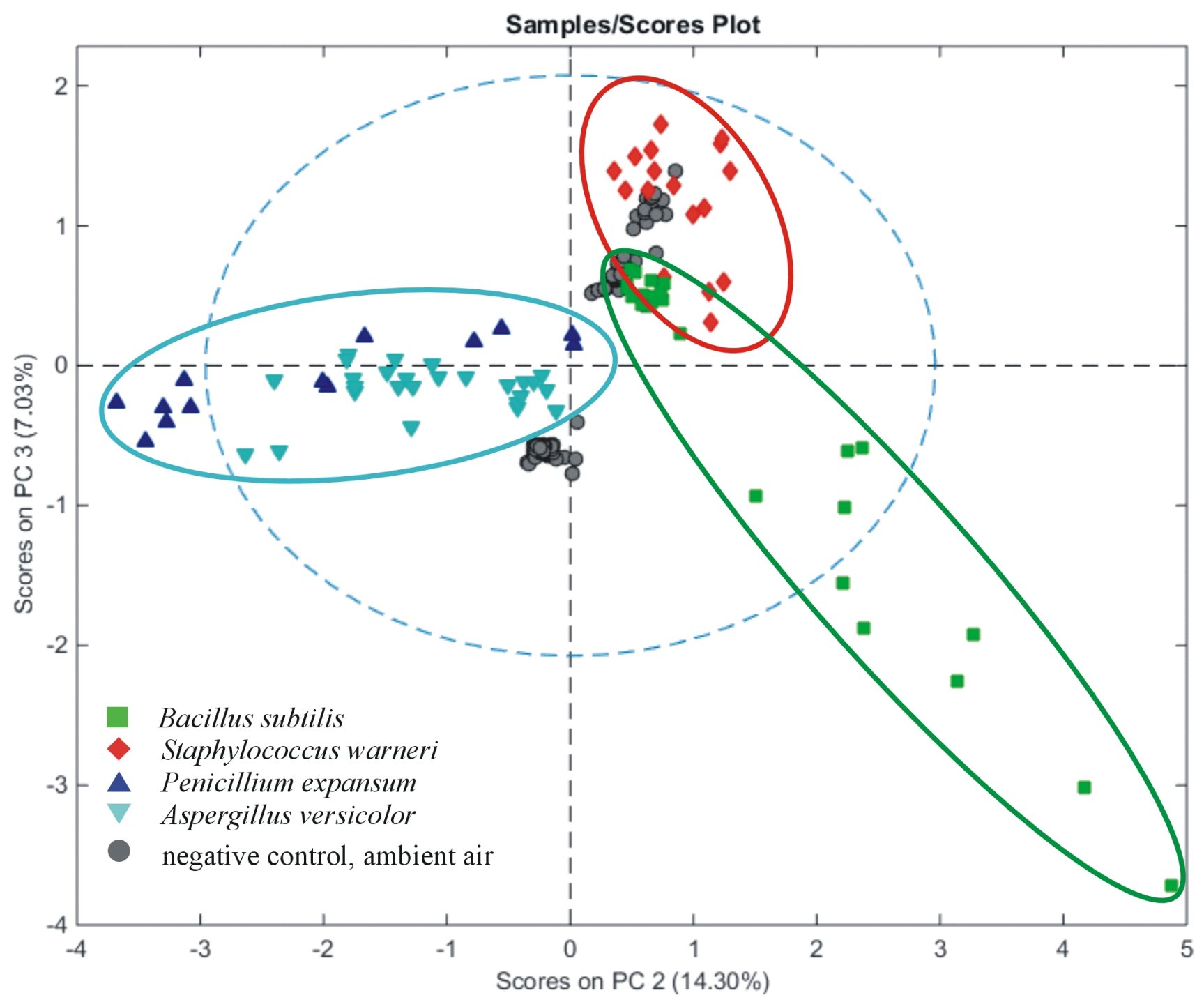

Figure 10. PC-2/3 score plot of 'E-Nose' measurements. The small grey clusters close to the origin derive from negative control experiments with ambient air.

variables as discriminant functions. The second part with 93 measurements was used as a prediction database. Performing the classification of PLSDA, the prediction database samples were classified with the model that was generated with the calibration dataset. The results of the PLSDA prediction are displayed in Tables 2 and 3. Table 2 shows the confusion matrix that gives an insight into the quality of classification. Concerning for example the culture S. warneri, $95.24 \%$ of positive S. warneri measurements are identified as such. Only $2.78 \%$ of the measurements are identified as false positive and $4.76 \%$ as false negative. The confusion table (Table 3) gives a closer insight on the misclassification of the specific species. It shows the correlation of actual measurement to the interpretation of the model as predicted class. It can be seen that no measurement of a target culture (positive sample) is being identified as another culture (also positive sample). Misclassifications only happen between positive and negative measurements. In detail, this means that a cultural sample is classified as clean sample and vice versa. If this error occurs to be too high, it could further be reduced by adapting the limit of detection threshold that correlates sensor signals and sensor signal noise to notable signal intensity via the three sigma method. Other misclassifications occur for negative control samples, but this is not surprising since sensor responses of negative samples are always very low in signal amplitude. 


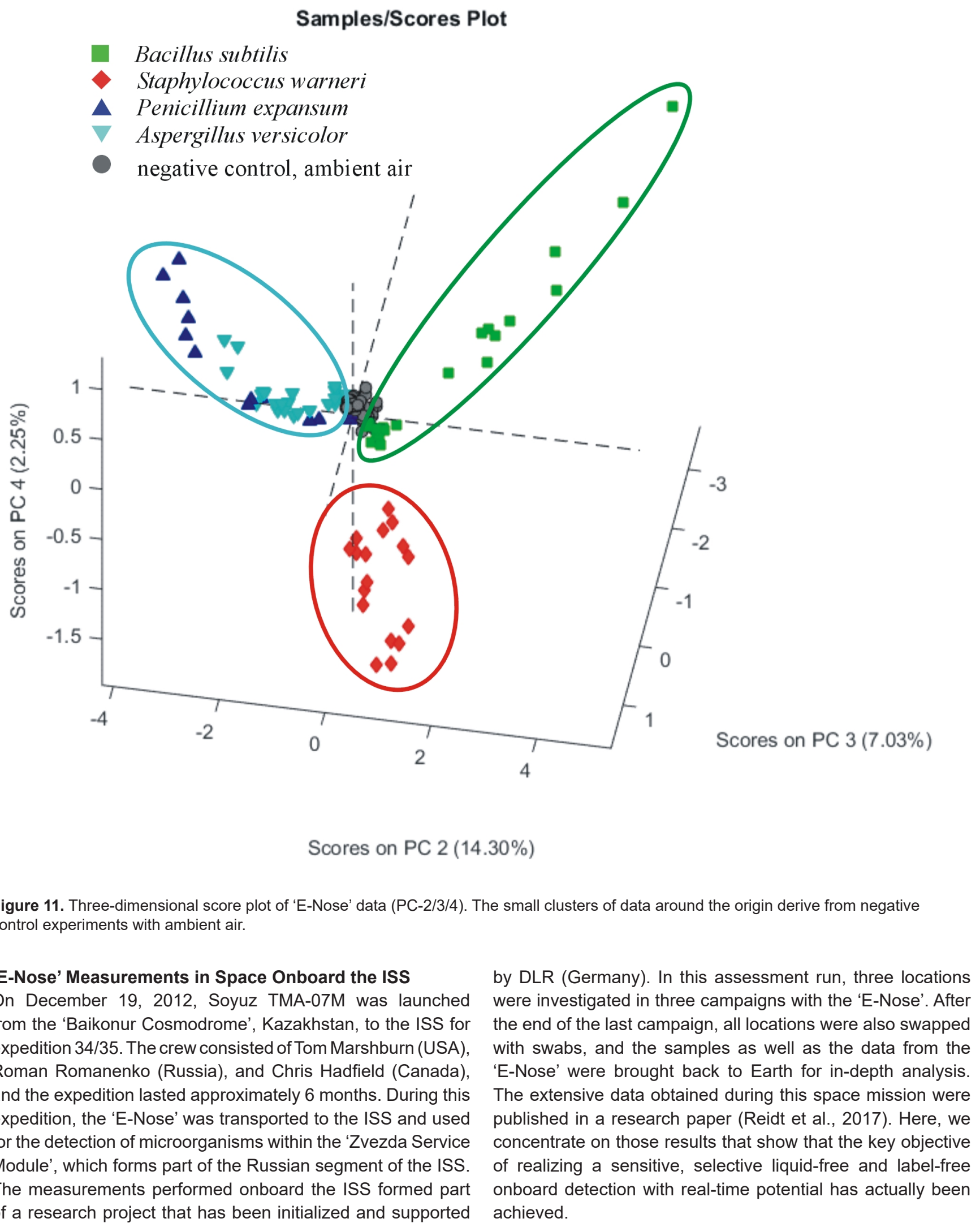




\section{Samples/Scores Plot}

Bacillus subtilis

Staphylococcus warneri

- Penicillium expansum

$\nabla$ Aspergillus versicolor

$\boldsymbol{\nabla}$ negative control, TSB

* negative control, Czapek-Dox

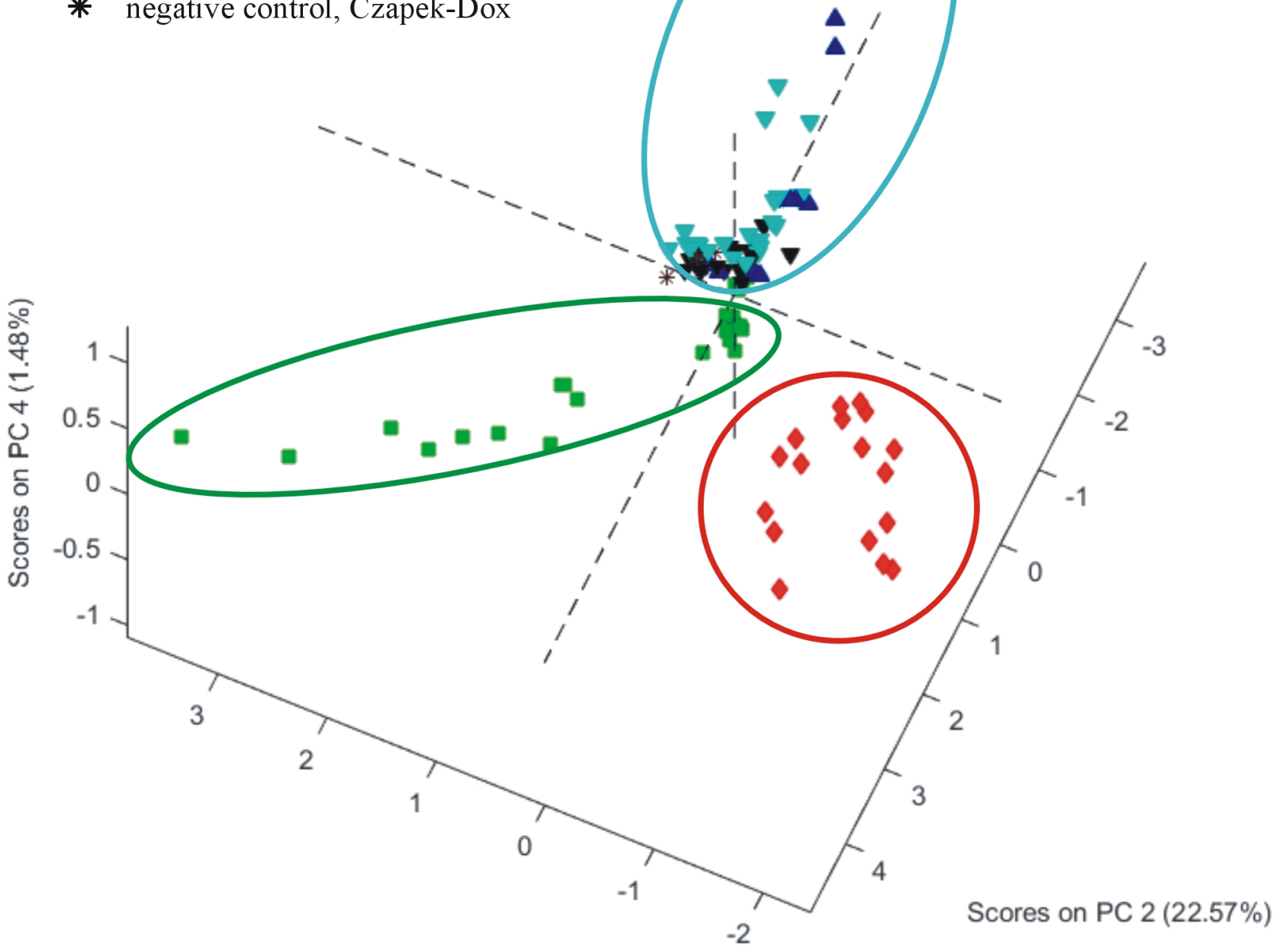

Scores on PC 3 (7.53\%)

Figure 12. Results diagrammed by three-dimensional PC (PC-2/3/4). Negative controls were done by culture media without organisms. Bacterial culture medium, Tryptic Soy Broth media (TSB): black triangles, Fungal culture medium, Czapek-Dox: black asterisk. 
Table 2: Confusion matrix; SW: Staphylococcus warneri, BS: Bacillus subtilis, PE: Penicilium expansum, AV: Apergillus versicolor, TSB: Tryptic Soy Broth, CZ: Czapek-Dox Agar

\begin{tabular}{ccccc}
\hline & $\begin{array}{c}\text { Correctly identified } \\
\text { positive }\end{array}$ & False positive & $\begin{array}{c}\text { Correctly identified } \\
\text { negative }\end{array}$ & False negative \\
\hline SW (TSB) & 0.95238 & 0.02778 & 0.97222 & 0.04762 \\
BS (TSB) & 0.85714 & 0.00000 & 1.00000 & 0.14286 \\
PE, AV (CZ) & 0.77778 & 0.00000 & 1.00000 & 0.22222 \\
Negative (CZ) & 0.66667 & 0.03333 & 0.96667 & 0.33333 \\
Negative (TSB) & 0.33333 & 0.12644 & 0.87356 & 0.66667 \\
Empty Airsampler & 0.75676 & 0.05357 & 0.94643 & 0.24324 \\
\hline
\end{tabular}

Table 3: Confusion Table; SW: Staphylococcus warneri, BS: Bacillus subtilis, PE: Penicilium expansum, AV: Apergillus versicolor, TSB: Tryptic Soy Broth, CZ: Czapek-Dox Agar

\begin{tabular}{ccccccc}
\hline & SW & BS & PE, AV (CZ) & Negative (CZ) & Negative (TSB) & $\begin{array}{c}\text { Empty } \\
\text { (TSB) Airsampler' }\end{array}$ \\
\hline Predicted as SW & 20 & 0 & 0 & 0 & 2 & 0 \\
Predicted as BS & 0 & 12 & 0 & 0 & 0 \\
Predicted as PE, AV & 0 & 0 & 7 & 0 & 0 & 0 \\
Predicted as (CZ) Negative & 0 & 0 & 1 & 2 & 2 & 0 \\
Predicted as (TSB) Negative & 0 & 2 & 0 & 0 & 2 & 0 \\
Predicted as Empty 'Airsampler' & 0 & 0 & 1 & 1 & 28 \\
\hline
\end{tabular}

Inspection of the data gathered onboard the ISS consistently concentrated in a cluster that did not overlap with the clusters of our still small and limited training set, indicating that the biocontamination onboard the ISS was dominated by another species not yet contained in our initial training set. Detailed microbiological analysis of the swabs brought back from the ISS revealed that the 'E-Nose' signals actually originated from the yeast Rhodotorula mucilaginosa. New training runs with $R$. mucilaginosa on Earth confirmed that this yeast does reproduce the data clusters obtained onboard the ISS. Overall, this first space mission showed that our sensor system hardware is able to perform under microgravity conditions and that it is able to detect the low levels of microbial contamination onboard the ISS. The limited match of the detected data patterns, however, clearly revealed the necessity to enlarge our presently small odor database through additional training runs on Earth to deal with the full complexity of bio-contamination onboard the ISS (Reidt et al., 2017).

\section{DISCUSSION}

The long experience in the field of manned space travel has shown that space flight conditions do cause dramatic decrements in the immune response of astronauts. The main reason for this physiological effect is the reduction of the lymphocyte proliferation in consequence of microgravity, psychosocial and physical stress, sleep disruption, high $G$ forces at launch and landing, radiation in space and nutritional factors (Ahearn et al., 1995; Cogoli, 1993; Sonnenfeld, 2003; Gueguinou et al., 2009). Astronauts therefore become more and more immunosuppressed in the course of long space travels. As a consequence, space crews are suffering considerable health risks when traveling in closed, contaminated environments. One important means of mitigating such risks is monitoring the growth of bacteria and fungi in space ships (Pierson, 2001; Taylor, 2015) and taking countermeasures in due time.

Hence, it is more than clear that astronauts need safe and sensitive detection systems for microorganisms onboard their spacecraft. Currently, onboard the ISS, microbiological investigations are done with classic sampling methods that rely on using swabs and contact plates, which will then have to be transported from the ISS down to Earth for accurate analysis in earth-bound laboratories (Novikova et al., 2006). This method is precise but has the disadvantage of long time delays between sampling and analysis and eventually requires countermeasures. Owing to the necessity of earthbased laboratory analysis, the currently employed method of space-borne microbial detection can no longer be pursued when long space mission as for instance flights to Mars are planned. 
The work performed in this investigation demonstrates that electronic noses equipped with arrays of MOX gas sensors are able to detect MVOCs emerging from microorganisms that occur inside the ISS and that such electronic noses allow the corresponding smell patterns to be separated into easily distinguishable clusters using PCA. Another encouraging aspect was the robustness of the MOX sensors with regard to sensor poisoning. As microorganisms emit a variety of compounds that could potentially poison the sensor surfaces, as for instance octane, 1-octene, isoprene, geosmin, alcohols, carboxylic acids, esters and sulfur derivatives [9] [11], it is encouraging that no signs of sensor poisoning could actually be observed for more than $2000 \mathrm{~h}$. Overall, therefore, our work presents a clear step forward to sensitive and selective detection systems for bacteria and fungi that are capable of operating under microgravity conditions inside space ships, that are easy to handle, and that allow data on the microbial contamination to be obtained in almost real time without earth-bound laboratories. As such systems do provide a fully local analysis of the microbial contamination inside a spaceship, the developed 'E-Nose' systems are useful for not only operation inside the ISS but also for much longer space missions, as for instance, flights to Mars.

Needless to say, the currently reached state of the art requires further development. The first obvious step is a massive expansion of the existing odor database. This step is necessitated by the fact that no less than 76 strains of bacteria and fungi are known to exist onboard the ISS (Novikova et al., 2009). This means that currently a large part of the microbiological flora is not yet determined by the current 'E-Nose'.

A massive increase in the size of the database also calls for a higher discrimination capability of the 'E-Nose' hardware. Considering the MOX sensor array configuration, an independent humidity sensor should be integrated to deal in a more satisfactory way with the strong and well-known humidity sensitivity of MOX gas sensors. The second obvious measure will be the optimization of the individual sensor operation temperatures to increase the selectivity of the array. If necessary, also other types of gas sensors and more sophisticated pattern recognition algorithms will have to be considered.

\section{CONCLUSIONS}

An electronic nose, featuring an array of ten MOX gas sensors, was modified to allow measurements of microbial contamination onboard the ISS under microgravity conditions.
For the training of the 'E-Nose', an odor database was set up consisting of two fungi and two bacteria strains, which are known to exist onboard the ISS. All strains were cultured on four different kinds of structural materials also used onboard the ISS.

It was demonstrated that the smell patterns produced by these strains form two autonomous clusters in an optimized PC score plot and the two kinds of fungi one single common large cluster.

Overall, our results demonstrate that it is possible to obtain in a facile way real-time data on the microbial contamination onboard the ISS. As a sample transfer between ISS and Earth is no longer required, the developed 'E-Nose' also qualifies for longer space missions, as for instance, flights to Mars.

Further research will be aimed at enlarging the existing odor database and enhancing the discrimination capabilities of the 'E-Nose' for future microbial detection tasks in the space.

\section{ACKNOWLEDGMENTS}

'E-Nose' is built under contract of the German Aerospace Center DLR e. V. with funds by the German Federal Ministry of Economic Affairs and Energy (50 PS 1402). In addition, we would like to thank Airsense Analytics, Germany, to use the diagram of the 'E-Nose'.

\section{REFERENCES}

Ahearn DG, Simmons RB, Price DL, Ajello L, Crow SA, Mishra SK, Pierson DL (1995) Fungal colonization of synthetic substrates for use in space craft. Journal of Industrial Microbiology 14, 26-30

Alam H, Saeed SH (2013) Modern applications of electronic nose: a review. International Journal of Electrical and Computer Engineering 3, 352-363

Baietto M, Wilson AD, Bassi D, Ferrini F (2010) Evaluation of three electronic noses for detecting incipient wood decay. Sensors 10 1062-1092

Bruins M, Bos A, Petit PL, Eadie K, Rog A, Bos R, van Ramshorst GH, van Belkum A (2009) Device-independent, real-time identification of bacterial pathogens with a metal oxide-based olfactory sensor. European Journal of Clinical Microbiology \& Infectious Diseases 28, 775-780

Cogoli A (1993) Space flight and the immune system. Vaccine 11, 496-503

Dalton P, Gelperin A, Preti G (2004) Volatile metabolic monitoring of glycemic status in diabetes using electronic olfaction. Diabetes Technology \& Therapeutics 6, 6534-6544 
Dutta R, Hines EL, Gardner JW, Boilot P (2002) Bacteria classification using Cyranose 320 electronic nose. BioMedical Engineering Online 1, 4

Gueguinou N, Huin-Schohn C, Bascove M, Bueb JL, Tschirhart E, Legrand-Frossi C, Frippiat JP (2009) Could spaceflight-associated immune system weakening preclude the expansion of human presence beyond Earth's orbit? Journal of Leukocyte Biology $\mathbf{8 6}$, 1027-1038

Juan Rodríguez J, Cristhian Durán C, Reyes A (2009) Electronic nose for quality control of colombian coffee through the detection of defects in "cup tests". Sensors 10, 36-46

Korpi A, Jarnberg J, Pasanen AL (2009) Microbial volatile organic compounds. Critical Reviews in Toxicology 39, 139-193

Korpi A, Pasanen AL, Pasanen P (1998) Volatile compounds originating from mixed microbial cultures on building materials under various humidity conditions. Applied and Environmental 64, 29142919

Marco AG (2012) Signal and data processing for machine olfaction and chemical sensing a review. IEEE Sensors Journal 12, 31893214

Novikova N, De Boever P, Poddubko S, Deshevaya E, Polikarpov N, Rakova N, Coninx I, Mergeay M (2006) Survey of environmental biocontamination on board the International Space Station. Research in Microbiology 157, 5-12

Novikova N, Pierson DL, Poddubko SV, Deshevaya Y, Ott CM, Castro VA, Bruce RJ (2009) Microbiology of the international space station. In U.S. and Russian Cooperation in Space Biology and Medicine (ed) pp. 263-278. American Institute of Aeronautics and Astronautics

Persaud K, Dodd G (1982) Analysis of discrimination mechanisms in the mammalian olfactory system using a model nose. Nature $\mathbf{2 9 9}$, 352-355

Pierson DL (2001) Microbial contamination of spacecraft. Gravitational and Space Biology Bulletin 14, 1-6

Reidt U, Helwig A, Plobner L, Lugmayr V, Kharin S, Smirnov Y, Novikova N, Lenic J, Fetter V, Hummel T (2017) Detection of microorganisms onboard the interntional space station using an electronic nose. Gravitational and Space 5, 89-111

Schaller E, Bosset JO, Escher F (1998) Electronic noses and their application to food. LWT - Food Science and Technology 31, 305-316

Sonnenfeld G (2003) Space flight, microgravity, stress, and immune responses. Advances in Space Research 23, 1945-1953

Su DY, Zhang GF, Zhang Y, Chen P, Zhang YJ, Zhu LY, Li J (2014) Electronic nose monitoring the Maillard reaction flavors of sesame oil from different production processes. Advance Journal of Food Science and Technology 6, 910-920

Taivans I, Bukovskis M, Strazda G, Jurka N (2014) Breath testing as a method for detecting lung cancer. Expert Review of Anticancer Therapy 14, 121-123

Taylor PW (2015) Impact of space flight on bacterial virulence and antibiotic susceptibility. Infection and Drug Resistance 8, 249-262
Wang P, Tan Y, Xie H, Shen F (1997) A novel method for diabetes diagnosis based on electronic nose. Biosensors and Bioelectronics 12, 1031-1036

Wilkins K, Larsen K, Simkus M (2000) Volatile metabolites from mold growth on building materials and synthetic media. Chemosphere 41, 437-446

Wilson A, Baietto M (2009) Applications and advances in electronicnose technologies. Sensors 9, 5099-5148 